\title{
On cross-modal similarity: Perceiving temporal patterns by hearing, touch, and vision
}

\author{
LAWRENCE E. MARKS \\ John B. Pierce Foundation Laboratory and Yale University, New Haven, Connecticut
}

\begin{abstract}
How does the similarity perceived between two brief temporal patterns depend on properties of the stimulus (durations of pulses and intervals between pulses) and on the sensory modality of presentation? Multidimensional scaling of similarity ratings obtained in a series of six experiments revealed four main findings: (1) Two-dimensional solutions provided good fits to all of the data, with the major dimension representing the order of intervals between pulses (long-short vs. short-long), the minor dimension representing overall duration (primarily of the pulses). (2) Essentially the same two-dimensional representation characterized similarity in intramodal comparisons (hearing, touch, vision) and cross-modal comparisons (hearing-touch, hearing-vision, vision-touch). But, (3) the major dimension-interval order-was even more prominent in crossmodal comparisons. Finally, (4) although individual subjects varied widely as to which dimension they weighted more heavily, individuals were consistent in this over all six (intramodal and cross-modal) tasks. Despite some differences between intramodal and cross-modal perception of similarity in temporal patterns, a large component of the similarity judgments transcends individual modalities.
\end{abstract}

What makes physically different temporal patterns appear alike? In trying to answer this general question, the present study evaluates the roles played by two properties of the stimulus: (1) the temporal characteristics of stimulation (pulse duration, interpulse interval), and (2) the sense modality through which the patterns are presented (hearing, touch, vision). In a nutshell, these experiments ask subjects to rate the degree of similarity/ dissimilarity between various pairs of temporal patterns when the two patterns are presented to the same modality and when each is presented to a different modality.

My primary concern here is the role played by different senses in the perception of temporal patterns. Two issues are at stake. The first one is: How do the (psychophysical) rules governing similarity in temporal-pattern perception compare in the ear, eye, and skin? The second is: How do these same modality-specific rules compare with the rules governing cross-modal pattern perception? Previous studies comparing modalities have typically focused not on judgments of similarity per se but on tasks of identification or discrimination. One question frequently asked, for example, is, How well can a person identify or distinguish two patterns, both presented either to the eye or to the ear? The answer is that auditory patterns are better recognized, more readily organized, and more accurately matched than visual ones (Fraisse, 1948; Garner \& Gottwald, 1968; Gault \& Goodfellow, 1938; Rubinstein \& Gruenberg, 1971). Discrimination of tactile patterns falls in between (Gault \& Good-

This research was supported by NIH Grant NS21326. I thank Shel Myers and Lisa Bogan for their assistance. Address correspondence to Lawrence E. Marks, John B. Pierce Foundation Laboratory, 290 Congress Avenue, New Haven, CT 06519. fellow, 1938). Sometimes, the question raised is: Are patterns distinguished as well in cross-modal comparison as they are in unimodal comparison? The answer here seems to be that cross-modal recognition of auditory and visual patterns is difficult when recognition of the visual patterns is difficult; but cross-modal recognition can be equivalent to intramodal recognition when the patterns are matched for difficulty and presented at relatively slow rates (fewer than 3 elements/sec) ( Rubinstein \& Gruenberg, 1971).

But, although they are related to those just mentioned, the questions I ask are somewhat different: Does the same set of psychophysical rules govern the perception of pattern similarity in different modalities-in the ear, eye, and skin? Moreover, does the same set of rules apply as well to cross-modal pattern perception? Jones (1976) has argued that rhythms, like melodies, are transposable. To the extent that this is so, relations among time intervals, not absolute values, should count the most. In a precisely transposable physical system, rhythmic similarity would be invariant across positive multiplicative transformation of stimulus durations; in a transposable psychophysical system, similarity would be invariant over positive multiplicative transformation of perceptual durations (which through psychophysical transformation could be nonlinearly related to physical durations). Either way, transposability probably lies at the very heart of temporalpattern similarity. Given that rhythms, or temporal patterns, can be transposed, it is important to determine whether the rules governing transposition are the same for auditory, tactile, and visual patterns.

The present study constitutes an early foray into this realm, although others have compared temporal processing per se in hearing, touch, and vision. Of these, the ear 
has the highest reputation, followed by the skin and then the eye (see Geldard, 1970). The same ordering of modalities appears in the discrimination of duration (Goodfellow, 1934), in the accuracy of judging temporal numerosity (Lechelt, 1975; cf. White \& Cheatham, 1959), and in the precision of synchronizing finger taps to auditory, tactile, and visual pulses (Kolers \& Brewster, 1985). The present studies enable us to see whether such an ordering is evident in perceived similarity of temporal patterns, that is, to see to what extent there is modal specificity in appreciating temporal patterns.

Because differences among the senses in temporal acuity can surely affect temporal-pattern perception, I tried, to a considerable extent, to minimize such effects here by using relatively slow patterns with few elements. Comparisons across the senses could also be influenced by the tendency to perceive auditory stimuli as lasting longer than visual stimuli of the same physical duration (e.g., Behar \& Bevan, 1961; Goldstone \& Goldfarb, 1963; Walker \& Scott, 1981). Ehrensing and Lhamon (1966) found perceived tactile durations to equal auditory ones. However, the findings of Craig (1973) - that when subjects adjusted the lengths of silent intervals between pulses to equal pulse duration, auditory intervals exceeded tactile intervals, which exceeded visual ones - could be interpreted to mean that tactile durations were intermediate in perceived length. In any case, the present study used identical sets of temporal patterns to investigate intramodal and crossmodal similarity. The aim of the study was to enable us to evaluate the extent to which similarities between temporal patterns presented to different modalities resemble similarities between patterns presented to the same modality: To what extent can people treat pattern information independently of the sensory modality of origin?

\section{METHOD}

\section{Apparatus}

All of the stimulus patterns were produced by a Commodore 64 microprocessor. Auditory stimuli were sawtooth waveforms, generated on the computer's sound interface device (SID) and presented through the speaker in the NAP monitor; the waveforms had a fundamental frequency of $220 \mathrm{~Hz}$ and a sound level of approximately $70 \mathrm{~dB}(\mathrm{~A})$ at the location of the subject's head. Tactile stimuli were also sawtooth waveforms produced by the computer's SID, with a fundamental frequency of $45 \mathrm{~Hz}$-low enough to minimize possible auditory cues when presented at comfortable levels, about $20 \mathrm{~dB}$ SL; vibrations were delivered to the index finger of the subject's right hand through a piston of $1.2 \mathrm{~cm}^{2}$ area, attached to a Brüel and Kjaer minishaker. Visual stimuli consisted of black rectangles (luminance, $8 \mathrm{~cd} / \mathrm{m}^{2}$ ), $0.7 \mathrm{~cm}$ high and $1.0 \mathrm{~cm}$ wide, presented against a pale green background $\left(40 \mathrm{~cd} / \mathrm{m}^{2}\right)$ at the center of the screen of the monitor and viewed at a distance of about $60 \mathrm{~cm}$.

Each pattern followed the scheme of pulse ${ }_{1}$-interval ${ }_{1}$-pulse $e_{2}$ interval $_{2}$-pulse $e_{3}$. There were two possible pulse durations, 0.1 and $0.2 \mathrm{sec}$, and four possible interpulse intervals, $0.15,0.25,0.45$, and $0.65 \mathrm{sec}$. Within a given pattern, the durations of all three pulses $\left(P_{1}, P_{2}\right.$, and $\left.P_{3}\right)$ were identical, but the interpulse intervals $\left(I_{1}\right.$ and $I_{2}$ ) were different, either 0.25 and $0.65 \mathrm{sec}$ or 0.15 and $0.45 \mathrm{sec}$, with the order of the interval durations varying from pattern to pattern. Eight different patterns were constructed in all (see Table 1).
Table 1

Durations of Pulses (P) and Interpulse Intervals (I) Used to Generate the Eight Temporal Patterns $\left(\mathbf{P}_{1}-I_{1}-P_{2}-I_{2}-P_{3}\right)$ in All Experiments

\begin{tabular}{|c|c|c|}
\hline $\begin{array}{c}\text { Interval } \\
\text { Duration (sec) } \\
I_{1}-I_{2}\end{array}$ & $\begin{array}{c}\text { Pulse } \\
\text { Duration (sec) }\end{array}$ & Pattern \\
\hline $.15-.45$ & $\begin{array}{l}.1 \\
.2\end{array}$ & $\begin{array}{l}1 \\
5\end{array}$ \\
\hline $.25-.65$ & $\begin{array}{l}.1 \\
.2\end{array}$ & $\begin{array}{l}2 \\
6\end{array}$ \\
\hline $.45-.15$ & $\begin{array}{l}.1 \\
.2\end{array}$ & $\begin{array}{l}3 \\
7\end{array}$ \\
\hline $.65-.25$ & $\begin{array}{l}.1 \\
.2\end{array}$ & $\begin{array}{l}4 \\
8\end{array}$ \\
\hline
\end{tabular}

Each could be paired with each other pattern in a given stimulus modality to make a total of 36 different pairs (the $8 \times 7 / 2=28$ combinations of different patterns, plus the 8 identity pairs), or in two modalities to make a total of 136 different pairs (the $36+36$ unimodal pairs, plus $8 \times 8=64$ cross-modal pairs). The goals in constructing the patterns were, first, to provide different pulse durations and interpulse intervals for subjects to judge, and second, to provide different sets of interval durations that would bear both similar and different ratios. As Table 1 indicates, for example, Pattern 6 is physically similar to Pattern 1 in that $I_{2}>I_{1}$ in both, although the pulse durations in Pattern 6 are twice as great and intervals are nearly twice as long.

\section{Procedure}

The procedure was essentially the same in all six experiments. Three experiments were unimodal (auditory, vibrotactile, and visual) and three were cross-modal (auditory-tactile, auditory-visual, and tactile-visual). The subject sat in front of the computer's monitor, on which the instructions appeared. Each trial contained two patterns, which the subject could present by pressing appropriate keys on the computer's keyboard. The subject could listen to, feel, or look at each pattern or both as many times as necessary before responding. When ready to make the response, the subject pressed a key and a rating line appeared on the screen. The rating line was $20 \mathrm{~cm}$ long, located at the center of the screen, and anchored at the left end by the label very, very different and at the right end by very, very similar. Appropriate keys permitted the subject to move a diamond-shaped marker to the proper location on the rating line to indicate the degree of similarity/dissimilarity between the two patterns. With a keystroke, the response's location was recorded (as one of 40 equally spaced positions, 0-39, on the line) and the next pair of stimuli prepared for delivery. Within a given unimodal session, the 36 pairs were presented in random order in four replications, making 144 trials in all. Within a given crossmodal session, the 136 pairs were presented once each in random order, and each subject served in two such sessions, held on different days. Order of pairs was random and different in every session for every subject.

The main experiments comprised the three unimodal and three pairwise cross-modal conditions. Initially, I planned to compare only hearing and vision, and therefore I originally asked the same 12 subjects to participate in the auditory, visual, and auditory-visual conditions. Subsequently, I decided to add comparisons of touch to touch, hearing to touch, and vision to touch. My aim was to have the same 12 subjects serve in all six conditions. However, 1 subject dropped out after completing the unimodal auditory, unimodal visual, and cross-modal auditory-visual experiments; he was replaced by another subject in the last three conditions. For purposes of replication, I report also a secondary series of experiments 
using 37 new subjects, 12 each participating in unimodal auditory and visual conditions and 13 participating in an auditory-visual condition in which they judged only the 64 cross-modal pairs.

The subjects were 6 young men and 6 young women in the main study, and 14 young men and 23 young women in the secondary series; they were all students or employees at local colleges, not selected for any special (e.g., musical) training or ability. (Although we identified 3 subjects of 12 in the main study and 4 of 10 in the secondary series as having had 5 or more years of musical lessons or training, such experience was unrelated to the individual differences evident in the results.)

\section{RESULTS AND DISCUSSION}

Similarity judgments were averaged across replications for each stimulus pair for each subject. A parsimonious and useful way to analyze and present the large amount of data is through multidimensional scaling. Jones (1976), for one, has argued that the time domain should be included-together with loudness and pitch-in a dimensional analysis of auditory perception. I chose for the present analysis a version of multidimensional scaling of individual differences (INDSCAL) that is fast and efficient, namely the program SINDSCAL (Pruzansky, 1975; Schiffman, Reynolds, \& Young, 1981; for a recent example of its application, see Clark, Carroll, Yang, \& Janal, 1986). Such multidimensional scaling assumes that the similarity judgments (actually, here their inversesdissimilarity judgments) represent the analogues to distances in a multidimensional, Euclidean space. SINDSCAL determines the structure of the space, that is, the location of the stimuli in it, characterizing performance of each group of subjects as a whole; within each group, differences among subjects in the weights that the subjects apply to each dimension enable the program to identify the orientation of the psychological axes or dimensions. Thus, no rotation of axes is necessary (or permissible without changing the meaning of the scaling solution). From a pragmatic point of view, the ease or readiness with which we can interpret the psychological meaning of the emergent dimensions becomes one criterion for accepting the very meaningfulness, if not the validity, of the approach. For my present purposes, multidimensional scaling was particularly valuable for two reasons: First, it enabled us to identify the psychological dimensions that presumably underlie the similarity judgments, and second, it readily permitted interindividual comparison.

Multidimensional scaling solutions were obtained for fits of the data to spaces of 2,3 , and 4 dimensions. All of the fits were good, with Dimension 1 accounting for the lion's share of the variance in all cases, the amount ranging from $43 \%$ to $69 \%$, depending on the condition; Dimension 2, orthogonal to Dimension 1, accounted for less-7\% to $34 \%$-but its contribution still was substantial. Dimensions 3 and 4 , by way of contrast, consistently contributed less than $10 \%$ each to the total variance. Moreover, unlike Dimensions 1 and 2, it was generally not possible to characterize in any simple way the nature of either Dimension 3 or Dimension 4; perhaps most salient here is the finding that, in the cross-modal experiments, neither Dimension 3 nor Dimension 4 separated the stimuli by modality. And finally, we can bring to bear a criterion of consistency: The ordering of stimuli in the main unimodal visual and auditory experiments was virtually identical to that in the corresponding secondary experiments on Dimensions 1 and 2, but different on Dimensions 3 and 4 . For these reasons, I shall present the results in terms of the two-dimensional solutions. Even if an occasional subject did include a third psychological component in his or her judgments of pattern similarity, two psychological dimensions do seem to suffice to capture the performance of the groups as a whole.

\section{Unimodal Similarity}

Two-dimensional structure. The upper panels of Figure 1 present the two-dimensional solutions for unimodal hearing, touch, and vision, respectively. (The main and secondary experiments on hearing and vision gave virtually identical results.) It is clear from inspection that the spatial representations of similarity among elements of this stimulus set are much the same in all three sense modalities. In each case, Dimension 1 dominates and corresponds to the order of the interpulse intervals: Dimension 1 places all of the patterns in which the order is shortlong into one group and the patterns long-short into another. This essential thythmic difference is clearly of paramount importance in determining the similarity of pairs of patterns, whether auditory, tactile, or visual.

If order of intervals-long-short or short-long-is all that matters to this dimension, then the fact that the ratio of the two longer durations is similar to that of the shorter durations implies that the points in each space should all line up in straight columns; that is, there should be only two scale values in Dimension 1. Although this is not quite the case, as the figures reveal, neither is there any systematic deviation from bipolarity across experiments. If, for example, the underlying psychophysical function relating perceived duration to physical duration is linear, then we might expect the patterns with the short interval durations to yield slightly more extreme values at both ends of Dimension 1, since the ratios at the short durations are more extreme. But this does not appear to be so. Instead, it appears that the subjects did largely treat the order of intervals categorically, as being either short-long or longshort. The outcome is in line with that of Fraisse (1946), who found that when subjects generated temporal patterns by tapping they tended to produce interpulse intervals with only two values, long and short, in ratios of about $2: 1$.

Dimension 2 counts less. And this is so for hearing, touch, and vision alike. The second dimension largely represents duration: It divides each set of stimuli into two groups, one containing the longer pulses, the other containing the shorter ones. Moreover, within each grouping of pulse durations, Dimension 2 separates longer from shorter pairs of interpulse intervals. Thus, Dimension 2 integrates the two stimulus parameters of pulse duration and interval duration into a single, composite psycholog- 


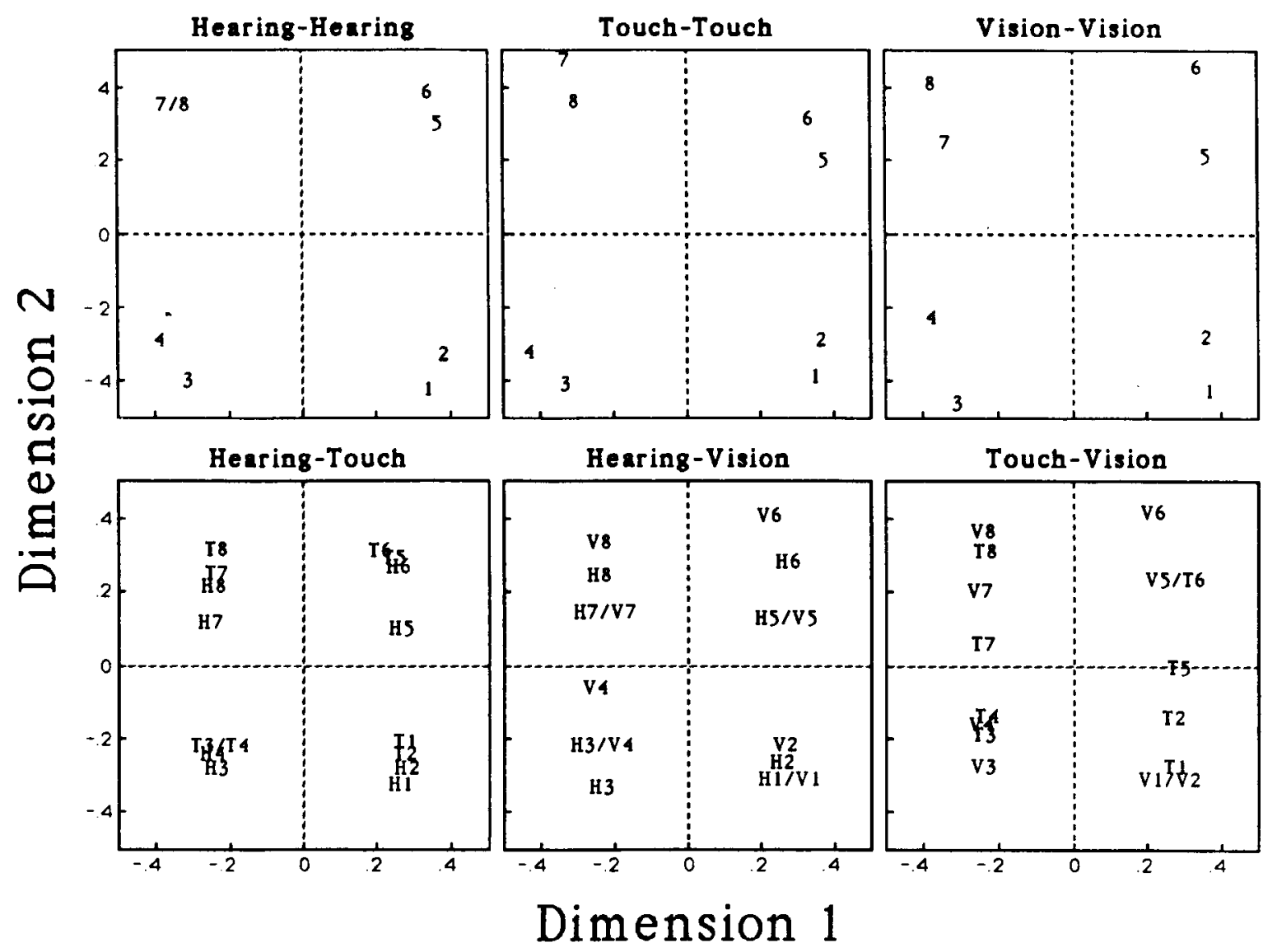

Figure 1. Multidimensional scaling solutions for unimodal (upper panels) and cross-modal (lower panels) temporal patterns. In each panel, Dimension 1 represents order of intervals (short-long, long-short); Dimension 2 represents increasing duration. Numbers refer to patterns as identified in Table $1 . H=$ hearing, $T=$ touch, and $V=$ vision.

ical dimension; but in doing this, it clearly gives much greater emphasis to the duration of the pulses. Despite the fact that the largest physical difference between durations of patterns contributed by the pulses $[3 \cdot(0.2-0.1)$ $=0.3 \mathrm{sec}$ ] equals the difference between the total durations of the intervals $[(0.65+0.25)-(0.45+0.15)=$ $0.3 \mathrm{sec}$, the average difference between scale values along Dimension 2 is nearly seven times as great for pulse duration as for interval duration. Note, furthermore, that the representation of this dimension, like that of Dimension 1 , is essentially independent of sensory modality.

Independence from modality. Are there any differences at all among modalities vis-à-vis the characteristics of the weightings of the two dimensions? Table 2 gives the percentages of variance for which each dimension accounts. Although results of the main experiments showed that Dimension 1 accounted for a substantially smaller fraction of the variance in tactile than in auditory or visual patterns, replication of the auditory and visual conditions with two new groups of subjects yields results commensurate with the results of the primary experiment with tactile stimuli. Nevertheless, as a general statement, given the fluctuations from experiment to experiment, it seems that Dimension 1 accounts for roughly
$50 \%-60 \%$ of the variance and Dimension 2 , for roughly $20 \%-30 \%$.

Recall that SINDSCAL derives the orientation of the axes in the multidimensional space from differences among subjects in the weightings attached to the presumed underlying dimensions. In the present experiment, the subjects showed marked individual differences; most subjects emphasized Dimension 1, the ordering of intervals, but a few emphasized Dimension 2, duration. That subjects tended to emphasize one or the other is shown by the negative correlations, over individuals, between the weightings on Dimensions 1 and $2(r=-.91,-.59$, and -.92 for hearing, touch, and vision, respectively, in the main experiments; $r=-.31$ and -.94 for hearing and vision in the secondary experiments). Of course, if the two dimensions accounted for $100 \%$ of the variance in every subject, the correlations would have to be perfect.

But what is most striking to me is the cross-modal consistency among individual subjects in the weightings on the two dimensions. Eleven subjects in the main experiments participated in all three unimodal tasks, and it is clear that each individual emphasized one dimension or the other regardless of whether the patterns were auditory, tactile, or visual. Correlation coefficients $(r)$ of the 
Table 2

Percentages of Variance-Accounted-For by Dimensions 1 and 2 (Multidimensional Scaling) in Unimodal and Cross-Modal Judgments

\begin{tabular}{lcc}
\hline & \multicolumn{2}{c}{ Percent Variance } \\
\cline { 2 - 3 } Sense Modality & Dimension 1 & Dimension 2 \\
\hline & Unimodal & \\
Hearing & $60.2(46.6)^{*}$ & $26.5(27.8)^{*}$ \\
Touch & 42.7 & 16.5 \\
Vision & $68.5(46.2)^{*}$ & $19.3(34.1)^{*}$ \\
Mean & 57.1 & 20.8 \\
& Cross-Modal & \\
Hearing-Touch & 54.2 & 9.8 \\
Hearing-Vision & 61.8 & 9.2 \\
Touch-Vision & 66.4 & 7.2 \\
Mean & 60.8 & 8.8 \\
& $\quad$ Unimodal Subsets & \\
Hearing (Hearing-Touch) & $58.7-M o d a l$ & 17.1 \\
Hearing (Hearing-Vision) & 58.7 & 16.0 \\
Touch (Hearing-Touch) & 60.8 & 18.1 \\
Touch (Touch-Vision) & 53.7 & 7.2 \\
Vision (Hearing-Vision) & 71.2 & 19.6 \\
Vision (Touch-Vision) & 56.8 & 16.5 \\
Mean & 57.4 & 15.8 \\
\hline
\end{tabular}

*Secondary experiments.

weightings on Dimension 1 equaled .71 (hearing-touch), .82 (hearing-vision), and .85 (touch-vision); on Dimension 2 , the values were $.84, .81$, and .65 , respectively. Each subject's reliance on either interval-order or duration transcended the particular sense modality stimulated.

In sum, the results for unimodal comparisons show that the multidimensional structure of perceptual similarity in simple temporal patterns-as inferred from multidimensional scaling solutions-is much the same in hearing, touch, and vision. The order of interval-duration between pulses counts most, whereas overall duration (mostly that of the pulses, but also of the intervals between pulses) counts less. Individuals differ in a consistent fashion, each placing greater or less weight on one dimension or the other, independently of the sensory modality through which the patterns are delivered.

\section{Cross-Modal Patterns}

Two-dimensional structure. The two-dimensional solutions for hearing-touch, hearing-vision, and touch-vision appear in the lower panels of Figure 1. Four primary features are evident:

First, the structure of multimodal perceptual space appears largely the same as-though perhaps not identical to-the structure of unimodal space. Dimension 1 again reveals the dominating effect of the order of interval durations; it splits the stimuli into two groups, one containing patterns with intervals short-long, the other containing patterns with the order of intervals reversed. Dimension 2 arranges stimuli by duration, combining duration of the pulses (more important) and duration of the intervals (less so). This, too, generally resembles the unimodal cases; cross-modally, however, interval dura- tion counts more relative to pulse duration than it does unimodally (one-fourth vs. one-seventh as much).

In the secondary hearing-vision experiment, the subjects judged cross-modal pairs but not unimodal pairs. Nevertheless, I was able to compare those results with the results obtained in the main experiment (eliminating from the latter the unimodal pairs) by treating each $8 \times 8$ cross-modal matrix as if modality were irrelevant and only temporal pattern mattered. Multidimensional scaling solutions again yielded two main dimensions, much like those obtained from the full $16 \times 16$ matrices of the main experiment.

Second, stimuli from different modalities can, in fact, be represented within a single multidimensional space. Thus, the two psychological dimensions-(1) temporal ordering of interpulse intervals and (2) duration-are crossmodal, perhaps amodal, characteristics. Particularly worth noting is the finding that modality per se did not appear as a dimension, not even when we carried the SINDSCAL solutions out to four dimensions. Thus, the subjects appear to have ignored any phenomenal difference between modalities. To the best of my knowledge, the only previous studies that attempted, through judgments of sensory stimuli, to identify dimensions in a perceptual similarity space common to different modalities are those of Wicker (1968), who explored similarities between colors (Munsell chips) and sounds (pure tones), and Marks (1985), who assessed similarities between pure tones heard and felt (as vibrations). In both of these studies, the unimodal and cross-modal judgments were obtained in different experimental sessions; hence, were modality a salient dimension of the underlying similarity space, it could not have appeared.

Although the present results give no indication of modality's being a perceptual dimension, modality may of course have a psychophysical effect in determining the exact spacings among stimuli. Because, for instance, pulses that are physically equal in duration may appear perceptually longer to the ear and skin than to the eye (e.g., Behar \& Bevan, 1961; Goldstone \& Goldfarb, 1963; Walker \& Scott, 1981), we might expect to find the perceived duration of auditory patterns to exceed that of visual patterns. Such an intermodal difference would appear as a displacement along Dimension 2 in the average location of one modality's stimuli from another's. In fact, Figure 1 reveals differences here-but not systematically in the expected direction-with auditory stimuli represented as being perceived a bit shorter than both visual stimuli (lower middle) and tactile stimuli (lower left) and visual and tactile as about equal (lower right). These differences, however, are relatively second order.

Unimodal versus cross-modal. This brings me to the third point: As examination of Table 2 indicates, Dimension 1 accounts for about $60 \%$ of the variance in the crossmodal scaling solutions. Viewed overall, these values approximate the percentages obtained from the unimodal solutions. Dimension 2, however, accounts for proportionally 
much less variance in the cross-modal experiments-in every case less than $10 \%$. The differences are statistically reliable, according to analyses of variance performed on individual weighting coefficients. There is a significant interaction between condition (unimodal vs. cross-modal) and dimension ( 1 vs. 2$)[F(1,10)=9.480, p=.01]$, in that the variance accounted by Dimension 1 is nearly constant $[F(1,10)=4.635, p=.054]$, whereas the variance accounted by Dimension 2 is greater unimodally $[F(1,10)$ $=15.31, p=.003]$. Hence, duration seems less important, less salient, in cross-modal comparisons than in unimodal comparisons of these temporal patterns.

This may need to be qualified: Perhaps it is the order of intervals per se that takes precedence-even when this dimension is not the most salient. Two subjects gave greater weighting to Dimension 2 than to Dimension 1 . If the cross-modal tasks merely induced subjects to weight to an even greater extent the more salient dimension, then those 2 subjects should have increased their weightings on Dimension 2 and reduced their weightings on Dimension 1; but, in fact, both of them-like the other subjectsactually increased their weightings on Dimension 1 and reduced them on Dimension 2.

To extend this line of analysis, I also compared judgments of intramodal similarity with judgments of crossmodal similarity by looking at subsets of data within each cross-modal experiment. Recall that each cross-modal condition in the main experiments contains within itself two complete sets of intramodal comparisons, since each condition pairs every stimulus with every other stimulus, both from the same modality and from the other modality. The hearing-touch condition, for example, contains hearinghearing, touch-touch, and hearing-touch pairings of stimuli. Thus, we can examine the multidimensional scaling solutions for (1) auditory patterns per se both within hearingtouch and within hearing-vision conditions, (2) tactile patterns per se within touch-hearing and touch-vision, and (3) visual patterns per se within hearing-vision and touchvision. In general, the resulting multidimensional scaling solutions resemble the solutions obtained in the pure single-modality conditions, containing the same two dimensions. And, as Table 2 indicates, the contributions of Dimensions 1 and 2 from the unimodal subsets of crossmodal data fall between the contributions obtained from the single modality conditions and those obtained from the cross-modal comparisons taken as a whole. [Although the differences in variance accounted for by Dimension 1 are not reliable, $F(2,20)=2.721, p=.09$, those in Dimension 2 are reliable, $F(2,20)=11.27, p<.001$, the unimodal subsets differing reliably on Dimension 2 from the overall cross-modal sets, $F(1,10)=18.49$, $p=.001$, though not from the pure unimodal sets, $F(1,10)=2.834, p=.12$.] By implication, this outcome suggests that the relatively small role of Dimension 2perceived duration-in the scaling solutions to overall cross-modal conditions reflects the subjects' similarity judgments given to the purely cross-modal subset of stimu- lus pairs, but not to the unimodal subsets. To the extent that this is so, cross-modal and within-modal judgments of temporal patterns were not wholly equivalent. Subjects used information about pulse (and interval) durations to a much greater extent when they compared patterns presented through the same modality than they did when they compared patterns presented through different modalities. Is it simplistic to suggest that the cross-modal task is more demanding than the unimodal task, and that, in response to the greater cognitive load, many subjects focused more on the salient dimensions of comparison?

Independence from modality. The fourth and final point concerns individual differences and individual consistencies. Again, as in the unimodal experiments, subjects varied in the degree to which they relied on one dimension or the other; the correlation between weighting coefficients for the two dimensions, over subjects, was -.71 for hearing-touch, -.83 for hearing-vision, and -.89 for touch-vision. And again, and more importantly, just as subjects were consistent from single modality to single modality in their weighting coefficients, so were they consistent across the bimodal tasks. For the 11 subjects who served in all conditions, intercorrelations in the cross-modal tasks averaged .97 for Dimension 1 and .89 for Dimension 2. Intercorrelations between weightings on the cross-modal and unimodal tasks averaged .88 for Dimension 1 and .81 for Dimension 2. This finding provides strong evidence for a large amodal component to perception of temporal patterns.

\section{Conclusion}

With the relatively slow temporal patterns used herepatterns that presumably place no overwhelming demand on the temporal discriminative ability of any of the modalities tested-perception of pattern similarity obeys a remarkably uniform set of psychophysical rules in hearing, touch, and vision. The overall structure of similarity space-the relations among the stimuli along the two dimensions of interval order and duration-remains strikingly uniform regardless of the modality stimulated. Moreover, the fact that individual subjects are consistent across modalities in their use of these dimensions suggests a supramodal strategy for evaluating, judging, and comparing these temporal patterns. To be sure, modalityspecific mechanisms probably exist in the brain for processing temporal discriminations (e.g., Swisher \& Hirsh, 1972); it is also probable that intersensory differences greater than those found here would emerge if the temporal discriminative capacity of the senses were challenged-with faster and more complicated patterns, for instance, the auditory system's superiority would surely be evident (cf. Garner \& Gottwald, 1968; Rubinstein \& Gruenberg, 1971). Nevertheless, when perceptual processing is relatively unencumbered by psychophysical limitations, it seems clear that people can and do employ a general, amodal strategy or strategiesperhaps parallel to modality-specific mechanisms-to 
judge temporal patterns quite independently of their sensory modality of origin.

\section{REFERENCES}

Behar, I., \& BeVAN, W. (1961). The perceived duration of auditory and visual intervals: Cross-modal comparison and interaction. American Journal of Psychology; 74, 17-26.

Clark, W. S., Carroll, J. D., Yang, J. C., \& Janal, M. N. (1986). Multidimensional scaling reveals two dimensions of thermal pain. Perception \& Psychophysics, 12, 103-107.

Craig, J. (1973). A constant error in the perception of brief temporal intervals. Perception \& Psychophysics, 13, 99-104.

Ehrensing, R. H., Lhamon, W. T. (1966). Comparison of tactile and auditory time judgments. Perceptual \& Motor Skills, 23, 929-930.

Fraisse, P. (1946). Contribution à l'étude du rhythme en tant que forme temporelle. Journal de Psychologie Normale et Pathologique, 39, 283-304.

Fraisse, P. (1948). Rhythmes auditifs et rhythmes visuels. L'Année Psychologique, 49, 21-41.

GARNER, W. R., \& GoTTWALD, R. L. (1968). The perception and learning of temporal patterns. Quarterly Journal of Experimental Psychology, 20, 97-109.

Gault, R. H., \& Goodfellow, L. D. (1938). An empirical consideration of audition, vision, and touch in the discrimination of temporal patterns and the ability to reproduce them. Journal of General Psychology, 18, 41-47.

GelDard, F. A. (1970). Vision, audition, and beyond. In W. D. Neff (Ed.), Contributions to sensory physiology (Vol. 4, pp. 1-17). New York: Academic Press.

Goldstone, S., \& Goldfarb, J. (1963). Judgment of filled and unfilled durations: Intersensory effects. Perceptual \& Motor Skills, 17, 763-774.

GoodFElLow, L. D. (1934). An empirical comparison of audition, vision, and touch in the discrimination of short intervals of time. American Journal of Psychology, 46, 243-258.
JONES, M. R. (1976). Time, our lost dimension: Toward a new theory of perception, attention, and memory. Psychological Review, 83, 323-355.

Kolers, P. A., \& Brewster, J. M. (1985). Rhythms and responses. Joumal of Experimental Psychology: Human Perception \& Performance, 11, 150-167.

LECHELT, E. C. (1975). Temporal numerosity discrimination: Intermodal comparisons revisited. British Journal of Psychology, 66, 101-108.

MARKs, L. E. (1985). Multidimensional scaling in the assessment of cross-modal similarity. In E. E. Roskam (Ed.), Measurement and personality assessment (pp. 165-177). Amsterdam: North-Holland.

PrUZANSKY, S. (1975). How to use SINDSCAL: A computer program for individual differences in multidimensional scaling. Murray Hill, NJ: Bell Telephone Laboratories.

Rubinstein, L., \& Gruenberg, E. M. (1971). Intramodal and crossmodal sensory transfer of visual and auditory temporal patterns. Perception \& Psychophysics, 9, 385-390.

Schiffman, S. S., ReYnolds, M. L., \& Young, F. W. (1981). Introduction to multidimensional scaling. New York: Academic Press.

SWISHER, L., HIRSH, I. J. (1972). Brain damage and the ordering of two temporally successive stimuli. Neuropsychologia, 10, 137-152.

WALKER, J. T., \& SCOTT, K. J. (1981). Auditory-visual conflicts in the perceived duration of lights, tones, and gaps. Journal of Experimental Psychology: Human Perception \& Performance, 7, 1327-1329.

White, C. T., \& Cheatham, P. G. (1959). Temporal numerosity: IV A comparison of the major senses. Journal of Experimental Psychology, 58, 441-444.

WICKER, F. W. (1968). Mapping the intersensory regions of perceptual space. American Journal of Psychology, 81, 178-188.

(Manuscript received November 3, 1986; revision accepted for publication April 3, 1987.) 